\title{
Concurrent anemia and stunting among schoolchildren in Wonago district in southern Ethiopia: A cross-sectional multilevel analysis
}

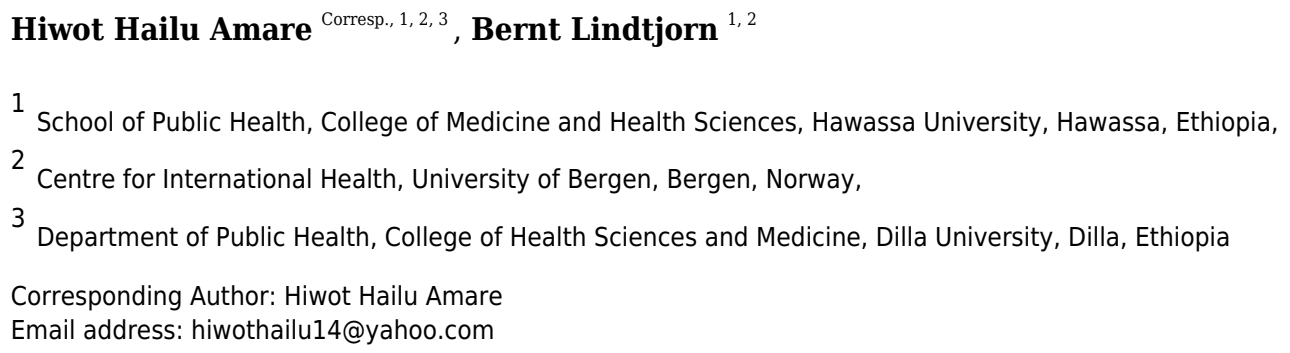

Background: Even if many schoolchildren in Ethiopia are anemic and stunted, few have studied the co-existence of anemia and stunting among schoolchildren in Ethiopia. In addition, multilevel analysis to explore the variation in prevalence of concurrent anemia and stunting (CAS) across schools and classes is rarely applied. Thus, we aimed to assess the prevalence and risk factors of CAS at the individual, household, and school level among schoolchildren in southern Ethiopia. Methods: We recruited 864 students aged 7-14 years from the Wonago district in southern Ethiopia using a three-stage random sampling, assigning four schools to level one, 24 classes to level two. We then randomly selected 36 children from each class, and recorded their weight, height, haemoglobin, intestinal helminthic infections, hygienic practices, dietary practices, household food insecurity, and socio-demographic information. A multivariate, multilevel logistic regression model was applied to detect potential risk factors for CAS. Results: The prevalence of CAS was 10.5\% (85/810) among schoolchildren, which increased with age in years (adjusted odds ratio [aOR] 1.39 [95\% confidence interval 1.13, 1.71, P=0.002]) and among children who always did not wash their hands with soap after use of latrine (aOR $4.30[1.21,15.3, \mathrm{P}=0.02])$. Children who walked barefoot (aOR $10.4[2.77,39.1, \mathrm{P}=0.001]$ ), were infected with Trichuris trichiura (aOR 1.74 [1.05, 2.88, $\mathrm{P}=0.03]$ ), or had head lice infestation (aOR 1.71 [1.01, 2.92, $\mathrm{P}=0.04]$ ) had higher CAS prevalence. Prevalence rates of CAS were low in those using treated drinking water (aOR $0.32[95 \% \mathrm{Cl} 0.11,0.97$, $\mathrm{P}=0.04]$ ). Most of the risk factors for CAS were identified at the individual level. The clustering effect measured by the intra-cluster correlation coefficient was $6.8 \%$ at school level and $19 \%$ at class. Conclusion: CAS prevalence is a moderate public health problem among schoolchildren in southern Ethiopia and varies across classes and schools. After controlling for clustering effects at the school and class levels, we found an association 
between CAS and increasing age, not always washing hands with soap after using latrine, walking barefoot, and $T$. trichiura infection. Using treated water for drinking was found to have a protective effect against CAS. Thus, educating children on personal hygiene and provision of safe drinking water could reduce the CAS burden in schoolchildren in rural areas of southern Ethiopia. 


\section{Concurrent anemia and stunting among schoolchildren in}

\section{Wonago district in southern Ethiopia: A cross-sectional}

\section{3 multilevel analysis}

\section{4}

5 Hiwot Hailu Amare ${ }^{1,2,3^{*}}$, Bernt Lindtjorn ${ }^{1,2}$

6

$7{ }^{1}$ School of Public Health, College of Medicine and Health Sciences, Hawassa University,

8 Hawassa, Ethiopia,

$9{ }^{2}$ Centre for International Health, University of Bergen, Bergen, Norway,

$10{ }^{3}$ Department of Public Health, College of Health Sciences and Medicine, Dilla University, Dilla,

11 Ethiopia 


\section{Abstract}

Background: Even if many schoolchildren in Ethiopia are anemic and stunted, few have studied the co-existence of anemia and stunting among schoolchildren in Ethiopia. In addition, multilevel analysis to explore the variation in prevalence of concurrent anemia and stunting (CAS) across schools and classes is rarely applied. Thus, we aimed to assess the prevalence and risk factors of CAS at the individual, household, and school level among schoolchildren in southern Ethiopia.

Methods: We recruited 864 students aged 7-14 years from the Wonago district in southern Ethiopia using a three-stage random sampling, assigning four schools to level one, 24 classes to level two. We then randomly selected 36 children from each class, and recorded their weight, height, haemoglobin, intestinal helminthic infections, hygienic practices, dietary practices, household food insecurity, and socio-demographic information. A multivariate, multilevel logistic regression model was applied to detect potential risk factors for CAS.

Results: The prevalence of CAS was 10.5\% (85/810) among schoolchildren, which increased with age in years (adjusted odds ratio [aOR] 1.39 [95\% confidence interval 1.13, 1.71, $\mathrm{P}=0.002]$ ) and among children who always did not wash their hands with soap after use of latrine (aOR $4.30[1.21,15.3, \mathrm{P}=0.02])$. Children who walked barefoot (aOR 10.4 [2.77, 39.1, $\mathrm{P}=0.001])$, were infected with Trichuris trichiura (aOR $1.74[1.05,2.88, \mathrm{P}=0.03])$, or had head lice infestation (aOR $1.71[1.01,2.92, \mathrm{P}=0.04])$ had higher CAS prevalence. Prevalence rates of CAS were low in those using treated drinking water (aOR 0.32 [95\% CI $0.11,0.97, \mathrm{P}=0.04]$ ). Most of the risk factors for CAS were identified at the individual level. The clustering effect measured by the intra-cluster correlation coefficient was $6.8 \%$ at school level and $19 \%$ at class. 
44 Conclusion: CAS prevalence is a moderate public health problem among schoolchildren in southern Ethiopia and varies across classes and schools. After controlling for clustering effects at the school and class levels, we found an association between CAS and increasing age, not always washing hands with soap after using latrine, walking barefoot, and T. trichiura infection. Using treated water for drinking was found to have a protective effect against CAS. Thus, educating children on personal hygiene and provision of safe drinking water could reduce the CAS burden in schoolchildren in rural areas of southern Ethiopia.

\section{Introduction}

Nearly half of all countries worldwide face the multiple burdens of malnutrition, including stunting, wasting, poor weight gain, and micronutrient deficiencies (International Food Policy Research Institute, 2015). Ethiopia has a school nutrition program designed to improve students' nutritional status (Federal Democratic Republic of Ethiopia Ministry of Education, 2012; Federal Democratic Republic of Ethiopia Ministry of Health, 2017). Despite school health and nutrition initiatives, a large proportion of schoolchildren in Ethiopia are still anemic or stunted (Federal Democratic Republic of Ethiopia Ministry of Education, 2012; Federal Democratic Republic of Ethiopia Ministry of Health, 2017). In Ethiopia, even though school access and enrolment have improved, about 10 million school-age children live in food-insecure areas (Federal Ministry of Education, 2015). Southern Ethiopia is among the regions in the country with inadequate school water supply (Federal Democratic Republic of Ethiopia Ministry of Education, 2017), and with poor sanitation practice with more than half of the schools still using open defecation (Grimes et al., 2017). 
Anemia affects about one-third of the population worldwide. Although the prevalence varies across different population groups and settings, the most common cause is iron deficiency (Kassebaum et al., 2014). Although there are few recent global reports for anemia among schoolaged children, WHO estimated in 2008 a 25\% prevalence among school-aged children (De Benoist et al., 2008). In 2016, the national prevalence of anemia among schoolchildren was $26 \%$ in Ethiopia, 20\% with iron deficiency anemia (Ethiopian Public Health Institute, 2016) and similar results are found in southern Ethiopia (Grimes et al., 2017; Shaka \& Wondimagegne, 2018). Stunting is also common among schoolchildren in southern Ethiopia with prevalence rates ranging from $15 \%$ to $57 \%$ (Bogale et al., 2018; Shaka \& Wondimagegne, 2018). Both anemia and stunting are preventable or treatable (Kakietek et al., 2017).

Studies indicate a link between anemia and stunting (Getaneh et al., 2017; Gutema et al., 2014). Stunting is often associated with anemia and recurrent infections among children (Gupta, 2017). Children with poor nutrient intake have weakened immunity, are susceptible to infections, and are at increased risk of anemia and long-term developmental delays (Bourke, Berkley \& Prendergast, 2016; Chaparro \& Suchdev, 2019). Despite these complex and interconnected health problems, few studies have investigated the co-existence of anemia and stunting in Ethiopia. Apart from the 2016 Ethiopian Demographic and Health Survey which demonstrated a prevalence rate of $24 \%$ of the co-morbidity of anemia and stunting among children aged under-5 years (Mohammed, Larijani \& Esmaillzadeh, 2019), we are not aware of similar studies on schoolchildren. However, several factors can contribute to the varied prevalence of anemia and stunting at both the individual (age, sex, nutritional deficiency, food consumption, poor hygiene, intestinal helminthic infections, parents' education) and household (wealth, family income and 
89 size, food insecurity, and access to hand-washing facilities) level (Assefa, Mossie \& Hamza,

90 2014; Getaneh et al., 2017; Gutema et al., 2014; Mesfin, Berhane \& Worku, 2015). Furthermore,

91 most previous studies did not consider the nested structure of school data (i.e., individuals nested

92 within the same class and classes nested within the same school) in their analysis. Few have

93 assessed the nutritional status of schoolchildren in rural southern Ethiopia (Grimes et al., 2017),

94 even if most school-aged children live in this rural area. This paper is part of a larger study on

95 school health problems that include the co-existence of anemia and stunting (CAS), intestinal

96 helminthic infections (Hailu Amare \& Lindtjørn, 2020), and skin problems in the Gedeo area of

97 southern Ethiopia. Therefore, we aimed to assess the prevalence and risk factors of CAS at the

98 individual, household, and school level among schoolchildren in the rural Wonago district in

99 southern Ethiopia.

Methods

\section{Study area, design, and participants}

The study was conducted in the Wonago district of the Gedeo zone in Southern Ethiopia. The district is $377 \mathrm{~km}$ south of Addis Ababa. The district has 17 rural and four urban kebeles, the smallest administrative units. In 2014, Wonago's population was estimated to be 143,989 people, with 1,014 people per square kilometer of land area. The district has 26 government health facilities (six health centers and 20 health posts), two private clinics, and two drug stores, along with more than 36,000 students in three urban and 22 rural primary schools. Most residents depend on cash crops of coffee, fruit, and ensete (Ensete ventricosum). 
111 We conducted this cross-sectional survey from February 2017 to June 2017. The study

112 population was schoolchildren and their parents or guardians. Students aged 7-14 years were

113 recruited in schools and their parents or guardians were contacted by visiting their homes.

\section{Sample size estimation}

116 Since this study was part of a large project aiming to identify school health problems, we

117 considered multiple factors to calculate the sample size using OpenEpi software (Sullivan, Dean

118 \& Soe, 2009) based on single population proportion (Daniel, 1999). Assuming a 95\% confidence

119 interval (CI), the maximum sample size was calculated using proportions of different variables

120 from previous studies (e.g., anemia [27\%] (Mesfin, Berhane \& Worku, 2015), stunting [30\%],

121 and thinness [37\%] (Mekonnen, Tadesse \& Kisi, 2013); intestinal parasites infections [27\%]

122 (Haftu, Deyessa \& Agedew, 2014), and skin tinea infection [50\%] (Ali, Yifru \& Woldeamanuel,

123 2009)); 5\% precision, and a design effect of 2 to account for multistage sampling.

124 To maximize the sample size, we used a prevalence of 50\%. After adding a $10 \%$ non-response

125 rate, we calculated a sample size of 845 schoolchildren.

126

127 This study targeted rural primary school-aged children. There are 22 rural primary schools in the

128 study district. Using a three-stage cluster sampling method, we randomly assigned 4 schools to

129 level one, 24 classes with 2,384 children to level two. We then randomly selected 36 children

130 from each class. When more than one child in a class was living together in the same household,

131 one of them was selected randomly by a lottery method. In total, we randomly recruited 864

132 schoolchildren. 
133 Children aged below 7 years and those older than 14 years were excluded from this study.

134 Children who had a physical deformity and serious illness could have been excluded from this

135 study, but we did not observe any of these cases during the data collection process.

136 The household of the student's parents or guardian was identified through a local guide. We

137 randomly selected and replaced participants who dropped out of school after the selection

138 process with participants from the same class, sex, and age by a lottery method. The recruitment

139 process is shown in Fig. S1.

\section{Outcome variables}

142 We assessed the prevalence and risk factors of concurrent anemia and stunting (CAS) among

143 schoolchildren. CAS among children was defined when a child was both anemic and stunted.

144 Anemia was recorded according to the WHO guidelines for school-aged children: haemoglobin <

$14511.5 \mathrm{~g} / \mathrm{dl}$ for those aged 5-11 years and $<12 \mathrm{~g} / \mathrm{dl}$ for those aged $12-14$ years. Anemia was

146 estimated using the haemoglobin value adjusted for altitude (WHO/NMH/NHD/MNM/11.1,

147 2011). Stunting among children was defined as height-for-age Z scores $<-2$ SD. Children with

148 height-for-age $\mathrm{Z}$ scores $\geq-3$ to $<-2 \mathrm{SD}$ were classified as moderately stunted, whereas those

149 with $<-3$ SD were classified as severely stunted (de Onis et al., 2007; WHO AnthroPlus for

150 Personal Computers Manual, 2009).

\section{Independent variables}

153 The independent variables at the individual level for the children were sex, age, hygienic

154 practices: nail trimming, hand washing with soap after latrine use, and walking barefoot; and 
155 dietary intake, meal habit before attending school (breakfast or lunch), reported illness in the

156 month prior to data collection, de-worming treatment in the past 6 months, and the presence of

157 Ascaris lumbricoides, Trichuris trichiura, hookworm, or head lice (pediculosis) infestations.

158 Parent individual level variables included the educational level of the mother and father.

Household factors included the wealth index, which was constructed using principal component analysis (Vyas \& Kumaranayake, 2006) based on household assets and included electricity, radio, television, mobile phone, table, chair, bed, separate room for kitchen, cooking place, land ownership, bank account, toilet facility, floor, and roof materials; family size; the level of food insecurity; using clean drinking water; and household access to food aid in the past 6 months. School factor included participation in the school meal programme.

\section{Data collection tools and procedures}

Ten trained enumerators conducted the interviews using a pretested and structured questionnaire that was adapted and developed in English from previous published literature (Alelign, Degarege \& Erko, 2015; Coates, Swindale \& Bilinsky, 2007; Gebreyesus et al., 2015; Haftu, Deyessa \& Agedew, 2014) and then translated into the local Gedeooffa language. Children were interviewed at their schools and parents at their homes.

173 Information related to the child's personal hygiene was collected from the children.

174 Measurements, such as weight, height, and haemoglobin, were taken from children in the school.

175 To measure weight, a digital portable scale (Seca 877, Seca GmbH, Germany) was calibrated to 176 the nearest $0.1 \mathrm{~kg}$. Children were weighed in light clothing and no shoes. To measure height, a 
177 measuring board (Seca 213, Seca GmbH, Germany) was calibrated to the nearest $0.1 \mathrm{~cm}$.

178 Children were measured while standing barefoot with parallel feet, heels, buttocks, and

179 shoulders, with their heads held upright, the backs of their heads touching the measuring board,

180 and their hands hanging by their sides. Capillary blood samples were taken for haemoglobin

181 measurement by trained and experienced laboratory technicians using a HemoCue Analyser $\mathrm{Hb}$

182301 (Angelholm, Sweden).

183 Stool samples were collected, processed, and examined using standard procedures (Montresor et

184 al., 1998; WHO, 1991). Samples were collected at school in the early morning, and stored in

185 stool cups labelled with an identification code, name, sex, age, and date. The specimens were

186 transported in a cold-box to Dilla University Teaching and Referral Hospital, where Kato-Katz

187 and formalin-ether concentration (FEC) techniques were used to identify helminthic infections

188 (Montresor et al., 1998; WHO, 1991). The result of each helminthic species from two diagnostic

189 techniques was recorded separately. We also examined the child's hair for external parasites,

190 such as head lice.

191 Household information was collected from the children's parents or guardians during home visits

192 through interviews and observations of the housing conditions. Information related to socio-

193 demographic characteristics, household assets, household food insecurity, use of treated

194 drinking water, access to food aid, child dietary intake, and illness in the past month were

195 collected from the parents or guardians at each household. Information related to the habit of

196 taking a meal regularly before school (breakfast if the child attended school in the morning and

197 lunch if the child attended classes in the afternoon) and the reason for not eating, was also

198 collected from the parents. Information related to de-worming treatment was collected from 
199 parents by showing them the de-worming tablet and asking, "In the past 6 months, was your

200 child given any de-worming treatment?"

201 Dietary intake was measured using a 24-hour dietary recall method (Swindale \& Bilinsky, 2006).

202 The children's parents were asked to list the food items that the children consumed within the

203 past 24 hours, and the children were asked if they ate outside the home the day before the survey.

204 We calculated the child Dietary Diversity Score (DDS) using the following nine food groups:

205 cereals, roots, or tubers; vitamin A-rich plant foods; other fruits; other vegetables; meat, poultry,

206 fish; eggs; pulses or legumes; milk and milk products; and food cooked in oils or fats. The food

207 items were classified based on FAO definition (Kennedy, Ballard \& Dop, 2011). A value of 1

208 was recorded if any of the disaggregated food group items were consumed, and 0 if none of the

209 disaggregated food group items were consumed (Swindale \& Bilinsky, 2006). DDSs were

210 computed by summing the previously mentioned food groups, with the score ranging from 0 to

2119 , and the mean DDS calculated for each child.

213 We assessed household food insecurity based on a 4-week recall period using nine items of the

214 Household Food Insecurity Access Scale (HFIAS) (Coates, Swindale \& Bilinsky, 2007;

215 Gebreyesus et al., 2015): Q1) worrying about food, Q2) unable to eat preferred foods, Q3) eating

216 a limited variety of foods, Q4) eating unwanted foods, Q5) eating small meals, Q6) eating fewer

217 meals in a day, Q7) having no food of any kind in the household, Q8) going to sleep at night

218 hungry, Q9) going a whole day and night without eating anything. 'No' responses were coded as

2190 and 'Yes' responses as 1 . Responses for frequency of occurrence items are coded as 1 for

220 'rarely', 2 for 'sometimes', 3 for 'often'. Households with no affirmative responses or 1 for Q1

221 and 'No' responses for the other items were considered food secure, whereas those reporting 2 or 
2223 for $\mathrm{Q} 1$ or 1, 2, or 3 for Q2 or 1 for Q3 or Q4 and no affirmative responses for Q5 to Q9 were

223 considered mildly food insecure; 2 or 3 for Q3 or Q4 or 1 or 2 for item Q5 or Q6 and 0 for Q7 to

224 Q9 were considered as moderately food insecure; 3 for Q5 or Q6 or 1, 2, or 3 for Q7 to Q9 were

225 categorized as severely food insecure. The score was computed by summing the responses for

226 the frequency of occurrence for the nine items (range 0 to 27).

\section{Data quality control}

229 Training was provided for all personnel participating in data collection, supervision, and data 230 entry. To minimize potential bias and validate the measurement tools prior to data collection, a

231 pre-test was conducted on 42 primary school-aged children in other schools not selected for this 232 study. Hence, we conducted a reliability test to observe inter- and intra-observer variations and 233 ensure reproducibility for anthropometric measurements. Observers measured the weights and 234 heights of 10 children twice. The intra-class correlation coefficient was used to estimate the 235 reliability of the continuous measurements (McHugh, 2012) (intra-observer reliability for 236 weight: 0.94 [95\% CI 0.78, 0.98], and height: 0.94 [95\% CI 0.78, 0.98]; inter-observer reliability 237 for weight: 0.92 [95\% CI 0.81, 0.97], and height: 0.91 [95\% CI 0.78, 0.97]). More than 90\% of 238 the intra- and inter-observer measurements show good reliability (Koo \& Li, 2016).

240 To record an accurate age for the children, we cross-checked information from the school

241 registry with the age information provided by the children's parents. The supervisors checked 242 data for completeness and consistency. 


\section{Statistical methods}

244 Data were entered into a database using the double-entry system in Epi-data version 3.1

245 (EpiData, Odense Denmark, 2004). Inconsistencies were cleaned and missing values were

246 addressed before analysis. After validation, the data were exported to SPSS version 20 (IBM

247 Corp, 2011) and STATA 15 software (StataCorp LLC, College Station, TX, 2017) for analysis.

249 The primary focus of this paper is to identify risk factors for CAS among school children.

250 However, earlier papers have shown that CAS share risk factors for stunting and anemia among

251 younger children. To assess this possibility, we provide separate risk estimates for anemia and

252 for stunting as supplementary files. We have summarised the shared risk factors for CAS,

253 anemia, and stunting at the end of the results section.

254 Descriptive statistics, frequency, percentage, mean, range, and standard deviation (SD) were

255 calculated to describe relevant variables. The proportions were determined for categorical

256 variables in relation to CAS. We used WHO AnthroPlus 1.0.4 software to calculate height-for-

257 age, weight-for-age, and body mass index-for-age $Z$ scores according to the standard reference

258 for children aged 5-19 years (WHO AnthroPlus for Personal Computers Manual, 2009). The

259 WHO AnthroPlus software allows Z scores for weight-for-age to be calculated only for children

260 up to 10 years of age (de Onis et al., 2007; WHO AnthroPlus for Personal Computers Manual,

261 2009). Therefore, children aged $>10$ years were excluded from the estimation of underweight

262 prevalence.

263 Household asset variables were categorized and coded as either 0 for absent or 1 for present. A

264 wealth index was then constructed using principal component analysis (Vyas \& Kumaranayake, 
265 2006). The internal consistency of the 14 variables was determined using a Cronbach alpha of

2660.78 and Kaiser-Meyer-Olkin sampling adequacy measure of 0.8. Socioeconomic indicators

267 (poor, middle-class, and rich) were categorized based on the first component explaining 28.3\%

268 of the data variance, with an Eigen value of 4.1.

269 We used three data hierarchies: school level, class level, and individual level (child or parent).

270 Students were clustered within the same class, and classes were nested within schools. We

271 included school- and class-level data during the analysis, and assessed potential confounding and

272 effect modifications using multivariate, multilevel regression and stratified analysis. 'Washing

273 hands with soap after latrine use sometimes or not always and never' were all categorized under

274 'not washing hands always'. 'Walking barefoot always and sometimes' were all categorized

275 under 'walking barefoot always'. Children with no CAS and yes CAS were stratified by the

276 categories of T. trichiura and not washing hands always.

277

278 Prior to the multivariate regression, we checked the collinearity among independent variables.

279 Both a bivariate logistic regression without considering random effects, and a multilevel logistic

280 regression model with random school and class effects were applied for assessment of potential

281 risk factors.

283 Using a multivariate, multilevel logistic regression model, we identified individual-, household-, 284 and school-level factors that may contribute to CAS. Six separate models were constructed for

285 CAS. Model I (empty) had no covariate indicating whether to consider the random-effect model.

286 Independent variables with $\mathrm{P}<0.25$ in the bivariate multilevel logistic regression model were 
287 introduced concurrently in each model. The variables with $\mathrm{P}<0.25$ in each independent model

288 were also maintained in the final model. Covariate variables; sex and wealth were with $\mathrm{P}>0.25$

289 in the bivariate model were retained in the final model to control for confounding. The final

290 model for CAS included individual child (sex, age, hand washing with soap after latrine use,

291 walking barefoot, T. trichiura, and head lice), parent (mother's education), household (wealth

292 status, family size, using treated water for drinking, access to food aid in the past 6 months), and

293 school (participation in school meal programme) factors.

Results were calculated as crude odds ratios (cORs) and adjusted odds ratios (aORs) with 95\%

CIs. Independent variables with $\mathrm{P} \leq 0.05$ in the final model were considered significant. Model fitness was checked using -2 log likelihood (deviance) and the Akaike information criterion (AIC). The model with the lowest deviance and AIC value was used as the final model (Twisk, 2006). A receiver operating characteristic (ROC) curve or area under the curve (AUC) was used to compare simple regression models with models considering a random effect. An AUC $\geq 0.7$ was considered acceptable (Merlo et al., 2016). In this study, the AUC for the CAS model considering a random effect was $>0.80$, whereas the AUC with a simple regression model was $<$ 0.7, suggesting that the model with a random effects is better than the model with a simple regression model for this outcome. Homogeneity within clusters at the school- and class-level was measured using the intra-cluster correlation coefficient (ICC).

\section{Ethics statement}

308 The institutional review board of Hawassa (IRB/005/09) and Regional Ethical Committee of 
310 Department (2/572/110) and District Education Office provided a letter of permission

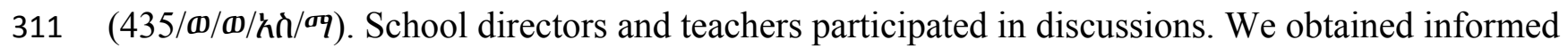

312 verbal (thumb print), and written (signed) consent from study participants' parents or guardians

313 and permission (assent) from children aged 12 years and older before the interviews. The

314 interviews and measurements were conducted in a private place for each participant.

315 Confidentiality of the participant's information was maintained. Children diagnosed as anemic

316 and who tested positive for intestinal helminthic infections were referred to the nearest health

317 institution for treatment according to standard guidelines (Drug Administration and Control

318 Authority (DACA) of Ethiopia, 2010).

\section{Results}

\section{Background information of study subjects}

322 The 861 schoolchildren (483 boys and 378 girls) in this study were aged 7 to 14 years, with mean [SD] age of 11.4 [1.9] years. All of the recruited children's parents or guardians participated in the household survey. The majority $(89.2 \% ; n=768)$ of children lived with their biological parents. The father's average age was 41 years and the mother's average age was 34 years. Among heads of households, 91.4\% (787/861) were men, and 8.6\% (74/861) were

327 women. The family size ranged from 3 to 14 persons (mean 6.7). Among parents, 88.4\% 328 (761/861) of mothers and 48.8\% (420/861) of fathers had never attended school. More than half 329 of the mothers $(54.0 \% ; n=463)$ were housewives, and most fathers $(77.4 \% ; n=619)$ were farmers. 330 Among the participants, 33.3\% $(\mathrm{n}=287)$ lived in poor households. Table $\mathrm{S} 1$ summarizes the 331 demographic and socioeconomic statuses of the schoolchildren and their parents. 


\section{Dietary habits and food insecurity}

334 The day before the survey, cereals, roots, or tubers $(100 \% ; n=861)$ were consumed by all 335 children; vitamin A-rich plant foods by $9.9 \%(n=85)$; other fruits by $18.6 \%(n=160)$; other 336 vegetables by $22.6 \%(n=195)$; meat or fish by $4 \%(n=35)$; eggs by $17 \%(n=147)$; pulses or 337 legumes by $46.9 \%(n=404)$; milk and milk products by $15 \%(n=129)$; and food cooked in oil or 338 fat by $3.7 \%(n=32)$. Coffee or tea was also consumed by $87 \%(n=749)$ of children a day prior to 339 the survey. The median and interquartile range of DDS was 2 (1-3), with a range of 1 to 8 food 340 groups consumed. The food items comprising each food group are found in the Supplementary 341 Information (Table S2).

342 The median and interquartile range of HFIAS score was $2(0-6)$, and scores ranged from 0 to 19. 343 Among the 861 children, 50.7\% ( $\mathrm{n}=437 ; 245$ [56.1\%] boys, 192 [43.9\%] girls) lived in food344 insecure households; $29 \%(n=253)$ in mildly food insecure households, $18 \%(n=155)$ in 345 moderately food insecure households, and 3.5\% $(n=30)$ in severely food insecure households. 346 The household food-insecurity item responses are presented in the Supplementary Information 347 (Table S3).

348

\section{School sanitation and hygiene facilities}

350 All schools had no access to drinking water and handwashing facilities. In all schools there were 351 pit latrines covered with cement. However, we observed that there were defections on the latrine 352 floors, and no toilet paper were available in the toilets room. 
354 Prevalence and risk factors for CAS

355 The prevalence of CAS was $10.5 \%(n=85 / 810$ [95\% CI 8.4, 12.6] among schoolchildren; 9.6\%, $356 \mathrm{n}=44$ boys and $11.6 \%, \mathrm{n}=41$ girls (Table 1 , Tables S4 and S5). The results of the bivariate 357 analysis for CAS are provided in Table S6.

358 We observed a clustering effect at the school- and class-level for CAS prevalence. The 359 calculated ICC indicated that $6.8 \%$ of the variability in CAS among children was attributable to 360 school-level factors and 19\% to class-level factors (Table S7).

All significant variables in the bivariate were significant in the multivariate model (Tables S6 and S7). As shown in Table 1 and Table S7, in the multivariate analysis, the odds of CAS increased with increasing age $[\mathrm{aOR}] 1.39$ [95\% confidence interval $1.13,1.71, \mathrm{P}=0.002])$. Children who always did not wash their hands with soap after use of latrine (aOR 4.30 [1.21, $15.3, \mathrm{P}=0.02]$ ), children who always walked barefoot (aOR 10.4 [2.77, 39.1, $\mathrm{P}=0.001]$ ), children infected with $T$. trichiura (aOR $1.74[1.05,2.88, \mathrm{P}=0.03])$, or had head lice infestation (aOR 1.71 $[1.01,2.92, \mathrm{P}=0.04])$ had higher $\mathrm{CAS}$ rates. Children using treated drinking water at their houses (aOR 0.32 [95\% CI $0.11,0.97, \mathrm{P}=0.04]$ ) were less likely to be affected by CAS. However, we did not find significant differences between CAS and sex, nail trimming, infection with $A$. lumbricoides, mother's education, wealth, family size, access to food aid in the past 6 months, and participation in the school meal programme (Table S7).

374 The crude and adjusted odds ratio estimates for variables, such as hand-washing habit, and walking barefoot, were different. The association between CAS and washing hands with soap

376 after latrine use was confounded by T. trichiura infection (Table 1). In addition, the association 
377 between CAS and walking barefoot was also confounded by hand washing with soap after latrine

378 use (Table 1).

\section{Prevalence of anemia and stunting}

381 Anemia alone occurred among 29.6\% $(\mathrm{n}=240 / 810)$ and stunting occurred in $32.3 \%(\mathrm{n}=278 / 861)$

382 of children (Tables S4 and S5). Commonly shared risk factors for stunting and CAS include age, 383 not always washing hands with soap after use of latrine, and head lice infestation. Using treated 384 water for drinking was also found to have a protective factor against stunting and CAS (Tables 385 S8, S9 and S10).

\section{Discussion}

388

389

390

391

392

393

394

395

396

397
Based on a standard definition, CAS was found to be a moderate public health problem among schoolchildren in southern Ethiopia (De Benoist et al., 2008; de Onis et al., 2019). Increasing age, not always washing hands with soap after using latrine, walking barefoot, T. trichiura infection, and head lice infestation were found to be associated with CAS. Using treated water for drinking was found to have a protective effect against CAS. Stunting and CAS share some of the same risk factors, including age, not washing hands with soap after use of latrine, and head lice infestation. Using treated drinking water was also a common shared protective factor for stunting and CAS. Significant clustering effect was observed at the school- and class-level for CAS prevalence. 
398 The rate of CAS was $10.5 \%$ among schoolchildren. This rate is lower than the recent report of $39923.9 \%$ for Ethiopia (Mohammed, Larijani \& Esmaillzadeh, 2019), 21.5\% for India, and 30.4\%

400 for Peru (Gosdin et al., 2018) among children under the age of 5 years. These variations could be 401 due to different study settings and participant ages. Moreover, unlike most studies, we conducted 402 our study in rural areas, which could also have influenced prevalence rates.

403 Using age as a continuous variable, we observed significantly increased odds of CAS with 404 increasing age, as described previously in Ethiopia (Alelign, Degarege \& Erko, 2015; Bogale et 405 al., 2018; Tariku et al., 2018). This could be explained by the older school-age children are in the 406 transition period to puberty, which increases nutritional demands (Soliman, De Sanctis \& 407 Elalaily, 2014).

Our results revealed an increased risk of CAS among children who always not washed their hands with soap after using the latrine compared to children who always washed their hands. This finding is supported by previous studies (Gosdin et al., 2018; Mahmud et al., 2015). Good hygiene is important to avoid acquiring infections and, thus, avoid CAS caused by helminthic infections. Moreover, walking barefoot may partly be indicative of low socio-economic status and poor hygiene. Relative to children who never walk barefoot, children who always walk barefoot had 5-times the risk of CAS in the bivariate analysis; introducing the habit of hand washing in the multivariate analysis, the risk of CAS was 10-times higher, potentially indicating the confounding effect of hand washing. Furthermore, these findings should be interpreted with caution as the number of children who always washed hands with soap after the use of latrine 419 and walked barefoot were few in this study. 
421 Species-specific analysis of intestinal helminthic infections revealed that T. trichiura is

422 significantly associated with CAS. This finding was also consistent with the previous findings

423 (de Gier et al., 2016; Shang et al., 2010; Stephenson, Holland \& Cooper, 2000). The link between

424 intestinal helminthic infections and CAS could be due in part to the inflammatory reactions to

425 worm infection in the intestinal mucosa, resulting in a loss of appetite, reduced food intake, and

426 impaired iron absorption (Shang et al., 2010; Stephenson, Holland \& Cooper, 2000). No

427 significant association was found between CAS and hookworm infections, probably due to low

428 statistical power because the number of children with hookworm infection was low.

The significant effect of head lice infestation on risk of CAS may be associated with low socioeconomic status and poor hygiene (Liao et al., 2017). We found lower odds of CAS among children using treated drinking water, suggesting a protective effect against intestinal helminthic infections (Matangila et al., 2014; Strunz et al., 2014; Worrell et al., 2016). Wealth status was also associated with CAS among children (Mohammed, Larijani \& Esmaillzadeh, 2019), but we found no difference in any of these odds across poor, middle-class, and rich households, findings in agreement with Alelign et al. (Alelign, Degarege \& Erko, 2015). There could be other socioeconomic factors not investigated in this study.

In this study, neither dietary diversity nor household food security was associated with CAS. The lack of association may be due similarities in the dietary diversity score and mild food insecurity in the area. A study from Kenya reported that participation in a school meal programme lowers

441 the risk of anemia, and stunting (Neervoort et al., 2013). Consistent with the previous finding in 442 southern Ethiopia (Shaka \& Wondimagegne, 2018), our study did not find a significant 443 difference in the rates of CAS among children participating in a school meal programme and 
444 those children who did not participate, although the observed association could be described as

445 borderline significant $(\mathrm{P}=0.08)$. Only cereals were served at school; therefore, the food being

446 served to the children at school was deficient in micronutrients and may explain why we failed to

447 detect a significant difference.

449 The strengths of this study include: we used a large representative sample of schoolchildren and applied a multilevel, mixed-effect model to identify risk factors for CAS. We also reported the

451 risk factors for anemia, and stunting. We diagnosed helminthic infections using two standard

452 techniques, the Kato-Katz and formalin-ether concentration. Data were clustered. Thus,

453 individual within-school and within-class dependencies (similarities) were identified and

454 measured using intra-cluster correlation coefficient.

455 Our study has some limitations. First, we used a cross-sectional design; thus, causality between

456 the outcome and independent variables cannot be determined with certainty. Second, due to

457 homogeneity issues, most school factors in this study (e.g., sanitation and hygiene facilities at the

458 schools) were not modelled. Third, we did not measure serum ferritin levels to assess iron status,

459 and we did not assess the presence of malaria. Recording dietary information for consecutive

460 days and measuring the quantity of food consumed could have improved the dietary information.

461 Unfortunately, we collected dietary information using only 24-hour dietary recall, and we did not

462 measure the quantity of the food. Further study in the area should assess the micronutrient levels

463 in schoolchildren. Our findings related to food insecurity could also be affected by over- or

464 under-reporting on the HFIAS questions. Over-reporting could arise if responses were related to

465 intentions to get food aid. Under-reporting of the last three HFIAS questions could also occur

466 due to fear of disclosing severe food insecurity related to cultural perceptions (Kabalo et al., 
467 2019). The results of hand washing may be affected by self-reported bias. Children who had their

468 head shaved could have been affected by head lice, but we did not observe any with these

469 characteristics during the examination. However, head lice could have been under-estimated.

470 Furthermore, including only children at rural schools may affect the external validity of this

471 study. However, we think that most school-aged children attended school. Thus, the findings can

472 be generalized to rural school-aged children in the same region where culture and living

473 standards are similar.

\section{Conclusions}

476 Our findings suggest the need for more effective nutrition programmes to tackle CAS among

477 schoolchildren in rural areas of Ethiopia. Interventions that improve hygiene can reduce the

478 morbidity caused by intestinal helminthic infections. Provision of safe drinking water and

479 promotion of treated water could also reduce comorbidities in schoolchildren. School teachers

480 should work with health workers to provide health education about personal hygiene protection.

481 Therefore, individual-, household-, and school-level intervention activities must be integrated to

482 improve the health of schoolchildren.

\section{Acknowledgments}

484 We are grateful to the schoolchildren, parents, and guardians who participated in this study. We 485 also thank the data collectors, supervisors, Gedeo Zone Health Department, Wonago District 486 Education Office, school directors, and teachers. We are also grateful to the Ethiopian Public

487 Health Institute for providing the Kato-Katz template. We would like to thank Dilla University 
488 Teaching and Referral Hospital for providing us with a laboratory examination room. We are 489 deeply grateful to Hawassa University and the University of Bergen for their support.

490

\section{References}

492

493

494

495

496

497

498

499

500

501

502

503

504

505

506

507

508

509

510

Alelign T, Degarege A, and Erko B. 2015. Prevalence and factors associated with undernutrition and anaemia among schoolchildren in Durbete Town, northwest Ethiopia. Archives of Public Health 73(1): 34 DOI: 10.1186/s13690-015-0084-x.

Ali J, Yifru S, and Woldeamanuel Y. 2009. Prevalence of tinea capitis and the causative agent among schoolchildren in Gondar, North West Ethiopia. Ethiopian Medical Journal 47(4): 261-269.

Assefa S, Mossie A, and Hamza L. 2014. Prevalence and severity of anemia among school children in Jimma Town, Southwest Ethiopia. BMC Hematology 14(1): 3 DOI: $10.1186 / 2052-1839-14-3$.

Bogale TY, Bala ET, Tadesse M, and Asamoah BO. 2018. Prevalence and associated factors for stunting among 6-12 years old school age children from rural community of Humbo district, Southern Ethiopia. BMC Public Health 18(1): 653 DOI: 10.1186/s12889-0185561-Z.

Bourke CD, Berkley JA, and Prendergast AJ. 2016. Immune dysfunction as a cause and consequence of malnutrition. Trends in Immunology 37(6): 386-398 DOI: 10.1016/j.it.2016.04.003.

Chaparro CM, and Suchdev PS. 2019. Anemia epidemiology, pathophysiology, and etiology in low- and middle-income countries. Annals of the New York Academy of Sciences 1450(1):15-31 DOI: 10.1111/nyas.14092. 
511 Coates J, Swindale A, and Bilinsky P. 2007. Household Food Insecurity Access Scale (HFIAS)

512 for Measurement of Household Food Access: indicator guide (v.3). Washington, DC: Food

513 and Nutrition Technical Assistance Project, Academy for Educational Development.

514 Daniel WW. 1999. Biostatistics: A foundations for analysis in the health sciences. New York:

$515 \quad$ John Wiley \& Sons, Ltd.

516 De Benoist B, Cogswell M, Egli I, and McLean E. 2008. Worldwide prevalence of anaemia 517 1993-2005; WHO global database of anaemia.

518 de Gier B, Nga TT, Winichagoon P, Dijkhuizen MA, Khan NC, van de Bor M, Ponce MC,

519 Polman K, and Wieringa FT. 2016. Species-specific associations between soil-transmitted

520 helminths and micronutrients in Vietnamese schoolchildren. American Journal of Tropical

521 Medicine and Hygiene 95(1): 77-82 DOI: 10.4269/ajtmh.15-0533.

522 de Onis M, Borghi E, Arimond M, Webb P, Croft T, Saha K, De-Regil LM, Thuita F,

523 Heidkamp R, Krasevec J, Hayashi C, and Flores-Ayala R. 2019. Prevalence thresholds for

524 wasting, overweight and stunting in children under 5 years. Public Health Nutrition

525

526

527

528 22(1):175-179 DOI: 10.1017/S1368980018002434.

de Onis M, Onyango AW, Borghi E, Siyam A, Nishida C, and Siekmann J. 2007. Development of a WHO growth reference for school-aged children and adolescents. Bulletin of the World Health Organization 85(9): 660-667 DOI: 10.2471/blt.07.043497.

Drug Administration and Control Authority (DACA) of Ethiopia. 2010. Standard Treatment

530 Guideline for Health Centers. Addis Ababa, Ethiopia: The Drug Administration and 531 Control Authority (DACA) of Ethiopia.

532 Ethiopian Public Health Institute. 2016. Ethiopian national micronutrient survey report. Addis 533 Ababa, Ethiopia: Ministry of Health. 
534 Federal Democratic Republic of Ethiopia Ministry of Education. 2012. National School Health 535 and Nutrition Strategy. Addis Ababa, Ethiopia.

536 Federal Democratic Republic of Ethiopia Ministry of Education. 2017. National school water

537 supply, sanitation and hygiene (SWASH) strategy and implementation action plan.

538 Federal Democratic Republic of Ethiopia Ministry of Health. 2017. School Health Programme

$539 \quad$ Framework. Addis Ababa, Ethiopia.

540 Federal Ministry of Education. 2015. Education Sector Development Programme V (ESDP V)

541 2015/16 - 2019/20: programme action plan. Addis Ababa, Ethiopia.

542 Gebreyesus SH, Lunde T, Mariam DH, Woldehanna T, and Lindtjørn B. 2015. Is the adapted Household Food Insecurity Access Scale (HFIAS) developed internationally to measure food insecurity valid in urban and rural households of Ethiopia? BMC Nutrition 1: 2. 2017. Prevalence of anemia and associated factors among schoolchildren in Gondar town public primary schools, northwest Ethiopia: a school-based cross-sectional study. PloS One 12(12): e0190151 DOI: 10.1371/journal.pone.0190151.

Gosdin L, Martorell R, Bartolini RM, Mehta R, Srikantiah S, and Young MF. 2018. The cooccurrence of anaemia and stunting in young children. Maternal \& Child Nutrition 14(3): e12597 DOI: 10.1111/mcn.12597.

Grimes JET, Tadesse G, Gardiner IA, Yard E, Wuletaw Y, Templeton MR, Harrison WE, and Drake LJ. 2017. Sanitation, hookworm, anemia, stunting, and wasting in primary schoolchildren in southern Ethiopia: Baseline results from a study in 30 schools. PLoS Neglected Tropical Diseases 11(10): e0005948 DOI: 10.1371/journal.pntd.0005948.

556 Gupta A. 2017. Nutritional anemia in preschool children. Singapore: Springer Nature, Pte Ltd. 
557 Gutema B, Adissu W, Asress Y, and Gedefaw L. 2014. Anemia and associated factors among

558 school-age children in Filtu Town, Somali region, Southeast Ethiopia. BMC Hematology

559 14(1): 13 DOI: 10.1186/2052-1839-14-13.

560 Haftu D, Deyessa N, and Agedew E. 2014. Prevalence and determinant factors of intestinal

561 parasites among schoolchildren in Arba Minch town, Southern Ethiopia. American Journal

562 of Health Research 2(5): 247-254 DOI: 10.11648/j.ajhr.20140205.15.

563 Hailu Amare H, and Lindtjørn B. 2020. Helminth infections among rural schoolchildren in

564 Southern Ethiopia: A cross-sectional multilevel and zero-inflated regression model. PLoS

$565 \quad$ Neglected Tropical Diseases 14(12):e0008002 DOI: 10.1371/journal.pntd.0008002.

566 International Food Policy Research Institute. 2015. Global nutrition report: actions and

567 accountability to advance nutrition and sustainable development. Washington, DC.

568 Kabalo BY, Gebreyesus SH, Loha E, and Lindtjørn B. 2019. Performance of an adapted

569 household food insecurity access scale in measuring seasonality in household food

570 insecurity in rural Ethiopia: a cohort analysis. BMC Nutrition 5(1): 54 DOI:

571 10.1186/s40795-019-0323-6.

572 Kakietek J, Eberwein JD, Walters D, and Shekar M. 2017. Unleashing gains in economic

573 productivity with investments in nutrition. Washington, DC: World Bank Group.

574 Kassebaum NJ, Jasrasaria R, Naghavi M, Wulf SK, Johns N, Lozano R, Regan M, Weatherall

575 D, Chou DP, Eisele TP, Flaxman SR, Pullan RL, Brooker SJ, and Murray CJL. 2014. A

576 systematic analysis of global anemia burden from 1990 to 2010. Blood 123(5):615-624

577 DOI: 10.1182/blood-2013-06-508325. 
578 Kennedy G, Ballard T, and Dop M. 2011. Guidelines for measuring household and individual 579 dietary diversity. Nutrition and Consumer Protection Division, Food and Agriculture 580 Organization of the United Nations. Rome, Italy: FAO.

581 Koo TK, and Li MY. 2016. A guideline of selecting and reporting intraclass correlation coefficients for reliability research. Journal of Chiropractic Medicine 15(2): 155-163 DOI: 10.1016/j.jcm.2016.02.012. H-T, and Fan C-K. 2017. Prevalence of Pediculus capitis in schoolchildren in Battambang, Cambodia. Journal of Microbiology, Immunology and Infection DOI: 10.1016/j.jmii.2017.09.003.

588

589

590

591

592

593

594

595

596

597

598

Mahmud MA, Spigt M, Bezabih AM, Pavon IL, Dinant G-J, and Velasco RB. 2015. Efficacy of handwashing with soap and nail clipping on intestinal parasitic infections in school-aged children: a factorial cluster randomized controlled trial. PLoS Medicine 12(6): e1001837e1001837 DOI: 10.1371/journal.pmed.1001837.

Matangila JR, Doua JY, Linsuke S, Madinga J, Inocêncio da Luz R, Van Geertruyden J-P, and Lutumba P. 2014. Malaria, schistosomiasis and soil transmitted helminth burden and their correlation with anemia in children attending primary schools in Kinshasa, Democratic Republic of Congo. PloS One 9(11): e110789-e110789 DOI:

10.1371/journal.pone.0110789.

McHugh ML. 2012. Interrater reliability: The kappa statistic. Biochemia Medica 22(3): 276282. 
599 Mekonnen H, Tadesse T, and Kisi K. 2013. Malnutrition and its correlates among rural primary 600 schoolchildren of Fogera District, Northwest Ethiopia. Journal Nutritional Disorders \& 601 Therapy: 2-7 DOI: 10.4172/2161-0509.S12-002.

602 Merlo J, Wagner P, Ghith N, and Leckie G. 2016. An original stepwise multilevel logistic 603 regression analysis of discriminatory accuracy: the case of neighbourhoods and health. 604 PloS One 11(4): e0153778 DOI: 10.1371/journal.pone.0153778.

605 Mesfin F, Berhane Y, and Worku A. 2015. Anemia among primary schoolchildren in Eastern 606 Ethiopia. PloS One 10(4): e0123615 DOI: 10.1371/journal.pone.0123615.

607 Mohammed SH, Larijani B, and Esmaillzadeh A. 2019. Concurrent anemia and stunting in 608 young children: prevalence, dietary and non-dietary associated factors. Nutrition Journal 609 18(1): 10 DOI: 10.1186/s12937-019-0436-4.

610 Montresor A, Crompton DWT, Hall A, Bundy DAP, and Savioli L. 1998. Guidelines for the 611 evaluation of soil-transmitted helminthiasis and schistosomiasis at community level : a 612 guide for managers of control programmes. Geneva: World Health Organization.

613 Neervoort F, von Rosenstiel I, Bongers K, Demetriades M, Shacola M, and Wolffers I. 2013.

614 Effect of a school feeding programme on nutritional status and anaemia in an urban slum: a 615 preliminary evaluation in Kenya. Journal of Tropical Pediatrics 59(3): 165-174 DOI: $616 \quad 10.1093 /$ tropej/fms070.

617 Shaka MF, and Wondimagegne YA. 2018. Anemia, a moderate public health concern among 618 adolescents in South Ethiopia. PloS One 13(7): e0191467 DOI:

619 10.1371/journal.pone.0191467. 
620 Shang Y, Tang L-H, Zhou S-S, Chen Y-D, Yang Y-C, and Lin S-X. 2010. Stunting and soil621 transmitted helminth infections among school-age pupils in rural areas of southern China.

622

623

624

625

626

627

628

629

630

631

632

633

634

635

636

637

638

639

640

641
Parasites \& Vectors 3: 97 DOI: 10.1186/1756-3305-3-97.

Soliman A, De Sanctis V, and Elalaily R. 2014. Nutrition and pubertal development. Indian Journal of Endocrinology and Metabolism 18(Supp1 1): S39-S47 DOI: 10.4103/22308210.145073.

Stephenson LS, Holland CV, and Cooper ES. 2000. The public health significance of Trichuris trichiura. Parasitology 121 Suppl: S73-S95 DOI: 10.1017/s0031182000006867.

Strunz EC, Addiss DG, Stocks ME, Ogden S, Utzinger J, and Freeman MC. 2014. Water, sanitation, hygiene, and soil-transmitted helminth infection: a systematic review and metaanalysis. PLoS Medicine 11(3): e1001620-e1001620 DOI: 10.1371/journal.pmed.1001620.

Sullivan KM, Dean A, and Soe MM. 2009. OpenEpi: a web-based epidemiologic and statistical calculator for public health. Public Health Reports 124(3): 471-474.

Swindale A, and Bilinsky P. 2006. Household Dietary Diversity Score (HDDS) for Measurment of Household Food Access: Indicator guide (v.2). Washington, DC: FHI 360/FANTA.

Tariku EZ, Abebe GA, Melketsedik ZA, and Gutema BT. 2018. Prevalence and factors associated with stunting and thinness among school-age children in Arba Minch Health and Demographic Surveillance Site, Southern Ethiopia. PloS One 13(11): e0206659 DOI: 10.1371/journal.pone.0206659.

Tezera R, Sahile Z, Yilma D, Misganaw E, and Mulu E. 2018. Prevalence of anemia among school-age children in Ethiopia: a systematic review and meta-analysis. Systematic reviews 7(1):80 DOI: 10.1186/s13643-018-0741-6.

PeerJ reviewing PDF | (2020:11:55273:2:0:NEW 25 Feb 2021) 
642 Twisk JWR. 2006. Applied multilevel analysis. A practical guides to biostatistics and 643 epidemiology. Cambridge: Cambridge University Press.

644 Vyas S, and Kumaranayake L. 2006. Constructing socio-economic status indices: how to use 645 principal components analysis. Health Policy and Planning 21(6): 459-468 DOI:

$646 \quad 10.1093 /$ heapol/czl029.

647 WHO. 1991. Basic laboratory methods in medical parasitology. Geneva: World Health $648 \quad$ Organization.

649 WHO AnthroPlus for Personal Computers Manual. 2009. Software for assessing growth of the 650 world's children and adolescents. Geneva: WHO.

$651 \mathrm{WHO} / \mathrm{NMH} / \mathrm{NHD} / \mathrm{MNM} / 1$ 1.1. 2011. Haemoglobin concentrations for the diagnosis of anaemia 652 and assessment of severity. Vitamin and Mineral Nutrition Information System. Geneva: 653 World Health Organization.

654 Worrell CM, Wiegand RE, Davis SM, Odero KO, Blackstock A, Cuéllar VM, Njenga SM, 655 Montgomery JM, Roy SL, and Fox LM. 2016. A cross-sectional study of water, sanitation, 656 and hygiene-related risk factors for soil-transmitted helminth infection in urban school, and 657 preschool-aged children in Kibera, Nairobi. PloS One 11(3): e0150744-e0150744 DOI:

$658 \quad$ 10.1371/journal.pone.0150744. 


\section{Table $\mathbf{1}$ (on next page)}

Bivariate and multivariate, multilevel, mixed-effect, regression analysis of CAS among schoolchildren in the Wonago district of southern Ethiopia, 2017 
1 Table 1 Bivariate and multivariate, multilevel, mixed-effect, regression analysis of CAS among

2 schoolchildren in the Wonago district of southern Ethiopia, 2017

\begin{tabular}{|c|c|c|c|c|c|c|c|}
\hline \multicolumn{2}{|l|}{ Variables } & \multicolumn{2}{|c|}{ CAS } & $\begin{array}{l}\text { Crude odds ratio } \\
\text { (COR) (95\% CI) }\end{array}$ & P-value & $\begin{array}{l}\text { Adjusted OR } \\
(95 \% \text { CI })\end{array}$ & P-value \\
\hline \multicolumn{2}{|l|}{ Individual child factors } & Yes (\%) & No (\%) & & & & \\
\hline \multirow[t]{2}{*}{ Sex } & Boys & $44(9.6)$ & $412(90.4)$ & $0.81(0.50,1.29)$ & 0.37 & $0.84(0.51,1.39)$ & 0.499 \\
\hline & Girls & $41(11.6)$ & $313(88.4)$ & 1.0 & & 1.0 & \\
\hline $\begin{array}{l}\text { Age in years } \\
\text { (continuous) }\end{array}$ & Mean (SD) & & & $1.36(1.13,1.65)$ & 0.001 & $1.39(1.13,1.71)$ & 0.002 \\
\hline \multirow{3}{*}{$\begin{array}{l}\text { Hand washing with } \\
\text { soap after use of } \\
\text { latrine }\end{array}$} & Always & $6(6.2)$ & $91(93.8)$ & 1.0 & & 1.0 & \\
\hline & $\begin{array}{l}\text { Sometimes } \\
\text { or not } \\
\text { always }\end{array}$ & $45(9.6)$ & $422(90.4)$ & $1.74(0.68,4.44)$ & 0.24 & $4.30(1.21,15.3)$ & 0.02 \\
\hline & Never & $34(13.8)$ & $212(86.2)$ & $1.67(0.58,4.81)$ & 0.34 & $3.10(0.82,11.8)$ & 0.09 \\
\hline \multirow[t]{3}{*}{ Walking barefoot } & Always & $6(30.0)$ & $14(70.0)$ & $5.36(1.74,16.5)$ & 0.003 & $10.4(2.77,39.1)$ & 0.001 \\
\hline & Sometimes & $40(10.5)$ & $341(89.5)$ & $1.16(0.70,1.91)$ & 0.57 & $1.18(0.68,2.05)$ & 0.55 \\
\hline & Never & $39(9.5)$ & $370(90.5)$ & 1.0 & & 1.0 & \\
\hline \multirow[t]{2}{*}{ Head lice } & Yes & $42(13.3)$ & $273(86.7)$ & $1.69(1.05,2.74)$ & 0.03 & $1.71(1.01,2.92)$ & 0.04 \\
\hline & No & $43(8.7)$ & $452(91.3)$ & 1.0 & & 1.0 & \\
\hline \multirow[t]{2}{*}{ T. trichiura } & No & $41(8.8)$ & $424(91.2)$ & 1.0 & & 1.0 & \\
\hline & Yes & $42(12.4)$ & $298(87.6)$ & $1.59(0.99,2.55)$ & 0.05 & $1.74(1.05,2.88)$ & 0.03 \\
\hline \multicolumn{8}{|l|}{ Household factors } \\
\hline \multirow{2}{*}{$\begin{array}{l}\text { Using treated drinking } \\
\text { water }\end{array}$} & Yes & $4(4.1)$ & $94(95.9)$ & $0.28(0.10,0.80)$ & 0.01 & $0.32(0.11,0.97)$ & 0.04 \\
\hline & No & $81(11.4)$ & $631(88.6)$ & 1.0 & & 1.0 & \\
\hline \multicolumn{8}{|l|}{ School factor } \\
\hline \multirow{2}{*}{$\begin{array}{l}\text { Participates in school } \\
\text { meal programme }\end{array}$} & No & $55(13.7)$ & $345(86.3)$ & 1.0 & & 1.0 & \\
\hline & Yes & $30(7.3)$ & $380(92.7)$ & $0.48(0.22,1.03)$ & 0.06 & $0.29(0.07,1.17)$ & 0.08 \\
\hline
\end{tabular}

3 CAS: concurrent anemia and stunting; CI: confidence interval; OR: odds ratio 